University of Texas at El Paso

ScholarWorks@UTEP

$3-2003$

\title{
Use of Fuzzy Expert's Information in Measurement and What We Can Gain from its Application in Geophysics
}

Leon Reznik

Vladik Kreinovich

The University of Texas at El Paso, vladik@utep.edu

Scott A. Starks

The University of Texas at El Paso, sstarks@utep.edu

Follow this and additional works at: https://scholarworks.utep.edu/cs_techrep

Part of the Computer Engineering Commons

Comments:

UTEP-CS-02-27a.

Published in Proceedings of the IEEE International Conference on Fuzzy Systems FUZZ-

IEEE'2003, St. Louis, Missouri, May 25-28, 2003, pp. 1026-1031.

\section{Recommended Citation}

Reznik, Leon; Kreinovich, Vladik; and Starks, Scott A., "Use of Fuzzy Expert's Information in Measurement and What We Can Gain from its Application in Geophysics" (2003). Departmental Technical Reports (CS). 357.

https://scholarworks.utep.edu/cs_techrep/357

This Article is brought to you for free and open access by the Computer Science at ScholarWorks@UTEP. It has been accepted for inclusion in Departmental Technical Reports (CS) by an authorized administrator of ScholarWorks@UTEP. For more information, please contact Iweber@utep.edu. 


\title{
Use of Fuzzy Expert's Information in Measurement and What We Can Gain from Its Application in Geophysics
}

\author{
Leon Reznik, Vladik Kreinovich and Scott A. Starks \\ Department of Computer Science, Rochester Institute of Technology, Rochester NY 14623-5608 and \\ Department of Computer Science and Pan-American Center for Earth and Environmental Studies \\ University of Texas at El Paso, El Paso, TX 79968-0518
}

\begin{abstract}
The paper considers the problem of measurement information fusion from different sources, when one of the sources is an information about approximate values of the measured variables or their combinations. The information is given with fuzzy models and is used in combination with the measurement results. The properties of the modified estimates are studied in comparison with the conventional ones. The conditions when an expert's information application can give a high gain are derived, the gain value is estimated, the recommendations to an expert on making predictions are given. The possible gain in measurement result efficiency in geophysical applications is analyzed.
\end{abstract}

\section{INTRODUCTION}

In many applications, often there is a need to fuse information coming from a variety of sources and having different uncertainties. In order to reach a high-quality decision in business, engineering and social applications nowadays one has to fuse information of different kinds (e.g. numerical, statistical, textual, visual, audio) from a variety of sources (e.g. engineering measurement systems, expert's opinions, images, sound tracks). Due to their nature, these sources differentiate in reliability and uncertainty of the information produced. Uncertainty also can be influenced by the characteristics of procedures and tools applied in information acquisition and processing.

This situation is typical for geological and geophysical sciences. For example, in the past fifteen years earthquake studies have grown from the collection of seismic data on frequency-limited seismometers (often in analog form) and mapping of surface faulting using geodetic techniques, to routine collection of digital seismic data on seismometers with broad frequency responses and mapping of surface deformation using a combination of geodetic, GPS and radar interferometry. For more detailed introduction into measurement problems in geophysical applications and especially into uncertainty estimation in measurement see [1].

The goal of this paper is four-fold. It attempts

to develop the more or less general method allowing fusing the predictions of the values of the measured variables and their linear combinations with the measurement results,

- $\quad$ to evaluate the uncertainty of the modified estimates,

- to evaluate the possible gain, which a prediction application can give us,

to analyze conditions when the prediction application might produce the strongest benefits.

\section{SOFT COMPUTING METHODS IN MEASUREMENT INFORMATION FUSION}

During a last few years one can witness an explosive rise of publications reporting fusion methodologies whose operation is based upon an application of some fuzzy and neural techniques. The numbers allow an introduction of some classification. The categorization given below does not pretend to be complete or comprehensive. It classifies examples of the soft computing applications in multi-source measurement information fusion and multi-sensor system design according to the goals achieved with their introduction.

1. Fuzzy logic and neural network approach to multisource data association and fusion. This group actually refers to multi-sensor systems and sensor arrays [2]. Within the group, one can find synergetic systems (mainly software, but some hardware could be included as in [3]), which intend to provide universally applicable solutions for fusion of the signals from different sources or for decision-making based on a variety of measurement results. Bloch [4] attempts to give a classification of numerical fusion operators which are applied to fuse imprecise, uncertain and incomplete information extracted from a variety of sensors with a degree of belief associated with each information source.

In [5] an extra knowledge is applied for choosing between different decisions obtained from solving conventional problems. In [6] the comprehensive methodology called Extended Logical Sensor Architecture (ELSA) was developed for constructing industrial sensor integration systems. ELSA has been developed for industrial applications, particularly, on-line grading and classification of non-uniform food products. It addresses a number of issues specific to an industrial inspection. The system is modular and scalable to accommodate new processes and changing customer demands. It is easy to understand so that non-expert users can construct, modify, and maintain the system. The sensor design methodology is based upon the object model, which represents object classifications through combinations of primary features weighted by fuzzy variables. The features guide the selection of sensors and processing routines; the classifications determine the rule base used by the inference engine for process decisions. Although inspection was the focus of this design, it is intended to become applicable in a variety of automation tasks, which may benefit from a multi-source perception system. 
Another multi-source fusion technique called Recurrent Fuzzy Inference (RFI) is presented in [7]. Here the membership functions of RFI are expressed by Radial Basis Function (RBF) with insensitive ranges. The shape of the membership functions can be adjusted by a learning algorithm. This algorithm is based on the steepest descent method and incremental learning, which can add new fuzzy rules.

A very interesting subsection of this group is composed by the multi-sensor systems trying to reproduce human capabilities of taste, odor and vision analysis. Besides improving accuracy, convenience and efficiency, the method allows realizing an engineering model of the sensing and recognizing systems of humans that combines all features of artificial olfactory and artificial taste. Rong et.al. [8] develop "electronic nose' and "electronic tongue" for wine classification based on fuzzy logic fusion technique. Llobet et.al. [9] describe an electronic nose based system, which employs an array of inexpensive commercial tin-oxide odor sensors, which have been used to analyze the state of ripeness of bananas. Readings were taken from the headspace of three sets of bananas during ripening over a period of 8-14 days. Lazzerini et.al. [10] present a new method for the fuzzy classification of odor samples that are obtained from an array of conducting polymer sensors. Linguistic expressions describing the response of both individual sensors and the sensor array to each chemical are derived from a fuzzy model of the sensor data.

2. Fuzzy logic and neural networks application for pattern recognition and classification. Here soft computing techniques are applied to make recognition more reliable as in [11], where a smart eddy-current sensor for locating and identifying metal tags used to recognize buried pipes or more accurate as in [12]. However, those systems should not be compulsory multi-source.

3. Fuzzy and neural methodology application for improving metrological and reliability characteristics. In this group fuzzy and neural methodology is commonly applied alongside with some extra (sometimes a priori) information available [13,14]. Su and Komata [15] consider an in-vehicletype load indicator and propose an error correction technique to compensate the error contained in the load measurement, by using fuzzy logic for dealing with changes in the loading states with a diversity of uncertainties. In [16] and [13] the object under measurement model which could be presented with neuro-fuzzy methods is applied for a sensor fault detection and even correction. Healy et.al. [16] describe a sensor in-range fault accommodation, which is a fundamental challenge of dual channel control systems in modem aircraft gas turbine engines. An on-board, real-time engine model can be used to provide an analytical third sensor channel that may be used to detect and isolate sensor faults. A fuzzy-logicbased accommodation approach is proposed that enhances the effectiveness of the analytical third channel in the control system's fault isolation and accommodation scheme. In [14] method the number of channels can be expanded and the sensor fault could be corrected. The similar approach [17] is applied for the validation of the measurement results in an ultrasonic sensor dedicated to mobile robot navigation
4. Sensors, which design is based on fuzzy neural network application. Here one can find a sophisticated design, incorporating the advanced soft computing techniques based on fuzzy neural networks application. In [18] and [19] two different networks, a feedforward neural network with an error backpropagation learning algorithm and a counterpropagation neural network, are employed to recognize the extracted features and provide a comparison of these two networks based on accuracy and speed. The data from multiple sensors are integrated through the proposed fuzzy logic model. Such a model is self-organizing and selfadjusting, learning from experience. Physical experiments of the metal cutting process are implemented to evaluate the proposed system.

Another example is [14], which develops a cascaded architecture of neural fuzzy networks with feature mapping (CNFM) to help the clustering of satellite sensor images. In the CNFM, a Kohonen's self-organizing feature map (SOFM) is used as a preprocessing layer for the reduction of a feature domain, which combines original multi-spectral gray values, structural measurements from co-occurrence matrices, and spectrum features from wavelet decomposition. In addition to the benefit of the feature space dimensional reduction, Kohonen's SOFM can remove some noisy areas and prevent the following training process from being overoriented to the training patterns, The condensed measurements are then forwarded into a neural fuzzy network, which performs supervised learning for pattern classification. The proposed cascaded approach is an appropriate technique for handling the classification problem in areas that exhibit large spatial variation and interclass heterogeneity (e.g., urban-rural infringing areas). The CNFM is a general and useful structure that can give us favorable results in terms of classification accuracy and learning speed.

\section{MATHEMATICAL PROBLEM FORMULATION}

A conventional way of solving the problem of measurement result estimation assumes its definition as a mathematical programming problem and search for the parameter $\mathrm{X}^{\prime}$ estimates by maximizing some criteria $\hat{X}=\max _{x} F\left(Y_{1}, Y_{2}, \ldots, Y_{n}, X\right)$, where $\mathrm{F}()$ is a functional, whose shape is determined by the estimation methods, $\mathrm{Yi}(\mathrm{i}=1, \mathrm{n})$ is a set of $\mathrm{m}_{\mathrm{i}}$ measurement results of the ith variable.

Let us consider a priori expert's information as a fuzzy constraint for the parameter vector $\mathrm{X}$ and given by the set of membership functions $\mu(f(X))$. The methods of an expert's information acquisition and its propagation through are discussed in [20]. In this case the estimation problem with a priori information application can be considered as an optimization problem with fuzzy constraints. By now research of fuzzy constraints has accumulated different methodologies of solving such problems. One of the simplest and the most obvious way is a unification of both functional criteria and constraints into one synergetic criterion and looking for a global solution as the optimization of such 
criterion. So the problem can be re-formulated as search for the estimate minimizing the synergetic criterion

$$
\tilde{\mathrm{X}}=\max _{\mathrm{X}} \mathrm{F}(\mathrm{Y} 1, \mathrm{Y} 2, \ldots, \mathrm{Yn}, \mathrm{X}) \times \mu(\mathrm{f}(\mathrm{X}))
$$

This problem could be tried with conventional or intelligent methods. We will call the solution of this optimization problem a modified estimate and apply it as an estimate of the measured value modified with the help of expert's information. The method choice should depend on the estimation techniques applied as well as on the membership function shapes.(see [21,22] for more detail).

\section{INVESTIGATION OF THE MODIFIED ESTIMATES IN COMPARISON TO THE CONVENTIONAL ONES}

\section{A. Properties}

Let us start our research from the most widely applied in practice the normally distributed measurement results (equation (1)) and the prediction, when the approximate value of a linear combination of a few variables is given (equation (2)),

(1) $\mathrm{Y}=\mathrm{AX}+\varepsilon \mathrm{y}$

(2) $b \approx B X$

where $Y$ is a $n \times 1$ vector (under the condition of $n>1$ ) of measurement results,

$\mathrm{X}$ is a $\mathrm{k} \times 1$ vector (under the condition of $\mathrm{k}>1$ ) of true values of the measurable variables,

Ey is a $n \times 1$ vector (under the condition of $n>1$ ) of measurement errors,

$b$ is is a $m \times 1$ vector (under the condition of $m>1$ ) of the forecast values,

A, B are matrices giving the structures of measurement and forecast schemes.

(1) could be considered as a standard measurement equation. Let us consider measurement results normally distributed with no bias and the covariation matrix $\Sigma y$.

(2) describes the forecast made that $m$ linear combinations of the measured variables (the combinations are given with the matrix B) approximately have values given by the vector $b$ components. The forecast is described mathematically by using the membership functions (see table 1) with the parameters of fuzziness given with the matrix $\Sigma$ b, which is a diagonal matrix with elements calculated as squares of the forecast fuzziness parameters.

With the direct measurements and predictions, matrices $\mathrm{A}$ and $\mathrm{B}$ become unit matrices and the equations (1) and (2) become simpler as

$\mathrm{Y}=\mathrm{X}+\varepsilon \mathrm{y}$

$b \approx X$

or in a case of one variable it would mean the prediction of an approximate value with the membership function of $\mu(x)=\exp \left(-(x-b)^{2} / \sigma^{2}\right)$.
Under general conditions, a conventional maximum likelihood estimate will be calculated as

$\hat{X}=\left(A^{T} \Sigma_{y}^{-1} A\right)^{-1} A^{T} \Sigma_{y}^{-1} Y$;

and a modified estimate should be calculated as

$\tilde{X}=\left(A^{T} \Sigma_{y}^{-1} A+2 B^{T} \Sigma_{b}^{-1} B\right)^{-1}\left(A^{T} \Sigma_{y}^{-1} Y+2 B^{T} \Sigma_{b}^{-1} b\right) ;$

or in one variable case

$\hat{X}=Y ;$ and

$\tilde{X}=\left(Y / \sigma^{2}+2 b / \delta^{2}\right) /\left(1 / \sigma^{2}+2 / \delta^{2}\right)=\left(Y+b / g^{2}\right) /\left(1+2 / g^{2}\right)$,

where $g=\delta / \sigma$ is the ratio of prediction uncertainty to measurement error, which is called later a prediction uncertainty factor.

The bias and the generalized dispersion of these estimates are equal correspondingly:

$$
M(\hat{X}-X)=0
$$

$$
\begin{aligned}
& \operatorname{cov}(\hat{X})=M\left[(\hat{X}-M \hat{X})(\hat{X}-M \hat{X})^{T}\right]=\left(A^{T} \Sigma_{y}^{-1} A\right)^{-1} \\
& M(\tilde{X}-X)=2\left(A^{T} \Sigma_{y}^{-1} A+2 B^{T} \Sigma_{b}^{-1} B\right)^{-1} B^{T} \Sigma_{b}^{-1}(b-B X) \\
& \operatorname{cov}(\tilde{X})=M\left[(\tilde{X}-M \tilde{X})(\tilde{X}-M \tilde{X})^{T}\right]= \\
& \left(A^{T} \Sigma_{y}^{-1} A+2 B^{T} \Sigma_{b}^{-1} B\right)^{-1} A^{T} \Sigma_{y}^{-1} A\left(A^{T} \Sigma_{y}^{-1} A+2 B^{T} \Sigma_{b}^{-1} B\right)
\end{aligned}
$$

where $\mathrm{M}()$ serves as a mean operator.

The dependence of a modified estimate's bias on the prediction uncertainty factor under different prediction errors in the case of one measured variable and one prediction made is given in fig.1. The enlarged section of this graph, which demonstrates the region where the bias becomes comparable or less than a measurement error is given in fig. 2. One may see that the bias becomes pretty small when the prediction error is still about 10 times higher than the prediction uncertainty. It means that the forecaster should be able to make reliable, practically unbiased predictions

\section{B. Bias of the modified estimates}

Statement 1. The modified estimate coincides with a conventional one if and only if the prediction value coincides with the conventional estimate.

Corollary 1. Modified estimate generally is biased against the conventional one.

Statement 2. Modified estimate lies between the conventional estimate and the forecast value.

Corollary 2. The modified estimate is shifted against the conventional one towards the forecast value.

However, the properties characterizing the accuracy of the modified estimate need to be investigated in order to evaluate a possible gain/lose.

1.Analyzing the formulae above one may see that a modified estimate's bias is proportional to the forecast error (b-BX)

2. One also can see that when a prediction is absolutely correct $(b-\mathrm{BX}=0)$ the modified estimate becomes unbiased.

3.On the other hand, the same result can be achieved when the fuzziness of the forecast is very big.

The modified estimate becomes unbiased when the forecaster makes a correct prediction or refuses to make any 
prediction at all. Actually, the bias of the modified estimate mainly depends on the ratio between the forecast error and the forecast fuzziness parameter or in other words on the ratio between the forecaster correctness and the forecaster confidence in the prediction made.

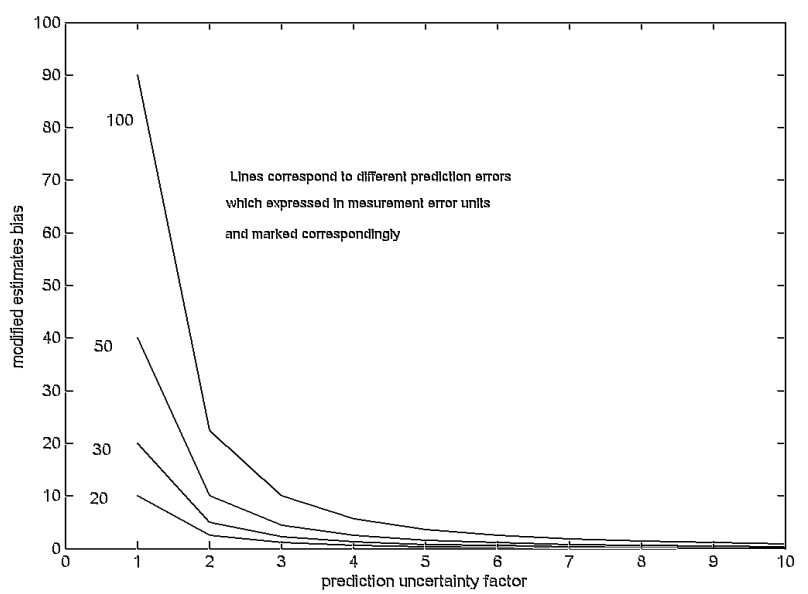

Fig.1 Relationship between the modified estimate's bias and the prediction uncertainty factor

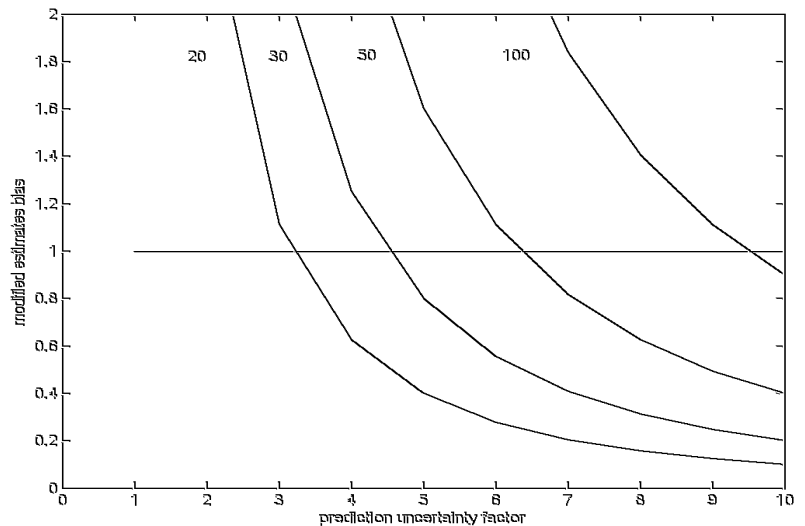

Fig.2 Relationship between the modified estimate's bias and the prediction uncertainty factor (enlarged section). The bold horizontal line marks the border where a bias becomes equal to a measurement error.

\section{Efficiency of the modified estimates}

Estimate's accuracy traditionally is taken as its dispersion. Comparing values (3) and (4) one may conclude that

1. when the forecast fuzziness is very big (practically the forecast is not given) the dispersions of estimates almost coincide with each other,

2. generally speaking the modified estimate's dispersion is smaller than the conventional one's, which means that the modified estimate is more efficient than the conventional one. It can be explained by the fact that the modified estimate is "pulled over" towards the forecast value.

However, despite this pleasant result the dispersion can not be taken as a good comparison base as the modified estimate could be biased due to the wrong forecast.
We have to consider another accuracy indicator, the mean square error (MSE), which is the mean of squares of deviations between the estimate and the true value. This indicator takes into account both the estimate's bias and its dispersion. MSE of the considered estimates will equal to:

$E_{\hat{X}}=M\left[(\hat{X}-X)(\hat{X}-X)^{T}\right]=\left(A^{T} \Sigma_{y}^{-1} A\right)^{-1}$;

$E_{\tilde{X}}=M\left[(\tilde{X}-X)(\tilde{X}-X)^{T}\right]=$

$\left(A^{T} \Sigma_{y}^{-1} A+2 B^{T} \Sigma_{b}^{-1} B\right)^{-1}\left(4 B^{T} \Sigma_{b}^{-1}(b-B X)(b-B X)^{T} \Sigma_{b}^{-1} B\right.$

$\left.+A^{T} \Sigma_{y}^{-1} A\right)\left(A^{T} \Sigma_{y}^{-1} A+2 B^{T} \Sigma_{b}^{-1} B\right)^{-1}$

The problem of this gain evaluation deserves a special consideration. To evaluate the gain provided by an expert's information application, let us choose the projection of the estimate's MSE, which can be written as

$T=\frac{1}{K} \operatorname{Sp}\left(E_{\hat{X}} E_{\tilde{X}}^{-1}\right)=$

$=\frac{1}{K} S p\left[\begin{array}{l}\left(A^{T} \Sigma_{y}^{-1} A\right)^{-1}\left(A^{T} \Sigma_{y}^{-1} A+2 B^{T} \Sigma_{b}^{-1} B\right) \\ \left(4 B^{T} \Sigma_{b}^{-1}(b-B X)(b-B X)^{T} \Sigma_{b}^{-1} B+A^{T} \Sigma_{y}^{-1} A\right)^{-1}\end{array}\right.$

$\left.\left(A^{T} \Sigma_{y}^{-1} A+2 B^{T} \Sigma_{b}^{-1} B\right)\right]$.

The gain depends on the measurement system and errors as well as prediction errors and its fuzziness (actually the ratio between the prediction error to the fuzziness). Figure 3 demonstrates the change in the gain value depending on the uncertainty prediction factor or actually on the prediction fuzziness when the mean measurement error is fixed. The maximum gain could be achieved when the prediction is absolutely accurate (prediction error is zero), that corresponds to the top line in fig 3 . Other lines show the relationship under the condition of some prediction error. This dependence on the prediction error is clearer in Figure 4, which demonstrates the gain change depending on it under different uncertainty prediction factors. One can see that with the increase in prediction errors the gain goes down and transforms into lose when the error in prediction becomes considerably bigger than its fuzziness.

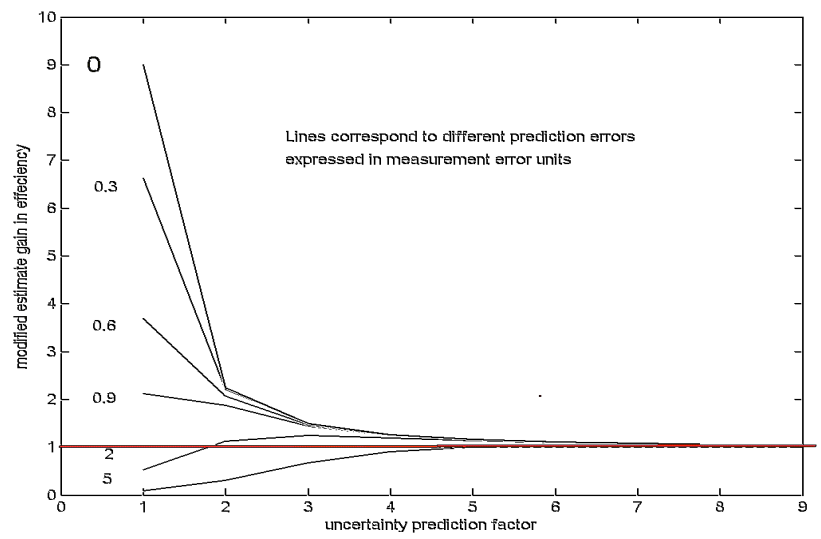

Fig.3. Relationship between the gain received by a modified estimate application and uncertainty prediction factor and prediction errors 
In a case of one measured value and one prediction the gain can be expressed as

$T=\sigma^{2}\left(1 / \sigma^{2}+2 / \delta^{2}\right)^{2} /\left(4(b-x)^{2} / g^{4} \sigma^{4}+1 / \sigma^{2}\right)$

or in terms of a prediction uncertainty factor

$T=\sigma^{2}\left(g^{2}+2\right)^{2} /\left(4(b-x)^{2}+g^{4} \sigma^{2}\right)$

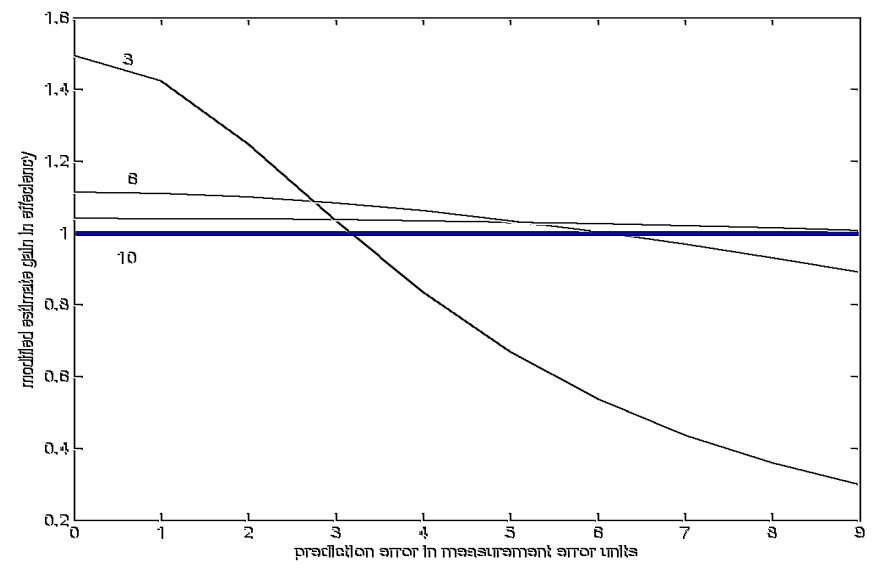

Fig.4. Relationship between the gain received by a modified estimate application and prediction errors and uncertainty prediction factor. Note: everything is measured in measurement error units

The biggest gain can be achieved with the correct predictions, i.e. $\mathrm{b}=\mathrm{BX}$, when

$T_{\max }=\operatorname{Sp}\left[\left(U+2\left(A^{T} \Sigma_{y}^{-1} A\right)^{-1} B^{T} \Sigma_{b}^{-1} B\right)^{2}\right]$, where $\mathrm{U}$ is a unit matrix

or in the case of direct measurements and predictions

$T_{\max }=\operatorname{Sp}\left[\left(U+2\left(\Sigma_{y} \Sigma_{b}^{-1}\right)^{2}\right]\right.$, from where one may see

that the maximum gain value depends on the ratio of the measurement errors to the prediction fuzziness. In another notation the maximum gain could be written as

$T_{\max }=\left(1+2 \sum_{i=1}^{K} \sigma_{i} / \sum_{i=1}^{K} \delta_{i}\right)^{2}$, which in the case of one

measured variable only becomes

$T_{\max }=(1+2 \sigma / \delta)^{2}$.

One may see that in order to increase the maximum gain, the prediction fuzziness should be decreased in comparison with the measurement errors. One may also conclude that if the prediction fuzziness is higher than 100 times measurement errors, the use of a priori information becomes doubtful as any possible gain value could be just a few percent. It means that one has to try to decrease the prediction fuzziness parameter. However, this strategy might be risky as it may result in loosing any gain at all.

\section{IS THERE ANY SENSE IN USE OF EXPERT'S INFORMATION AND UNDER WHICH CONDITIONS?}

\section{A. Mathematical point of view}

Let us try to clarify conditions when the modified estimate superiors a conventional one against the MSE indicator or becomes more accurate. Mathematically the condition
$E_{\tilde{X}}<E_{\hat{X}}$ can be shown equivalent to the condition

$B^{T} \Sigma_{b}^{-1}(b-B X)(b-B X)^{T} \Sigma_{b}^{-1} B$

$<B^{T} \Sigma_{b}^{-1} B+B^{T} \Sigma_{b}^{-1} B\left(A^{T} \Sigma_{y}^{-1} A\right)^{-1} B^{T} \Sigma_{b}^{-1} B$;

or in another notation

(5) $(b-B X)(b-B X)^{T}<\Sigma_{b}+B\left(A^{T} \Sigma_{y}^{-1} A\right)^{-1} B^{T}$.

The left side of this inequality constitutes the prediction error square, the right side combines the prediction fuzziness with measurement errors in squares also. One may see that in order to improve the accuracy of the estimate, the prediction error should be not bigger than its fuzziness. Actually, it may be even larger a bit, by some value, which depends on the measurement errors. This relationship becomes clearer in the case of direct measurements and predictions when the matrices $\mathrm{A}$ and $\mathrm{B}$ are unit matrices, and matrices $\Sigma_{b}$ and $\Sigma y$ are diagonals. In this case the condition (5) becomes more transparent as

(6) $\sum_{i=1}^{K}\left(b_{i}-x_{i}\right)^{2} / K<\sum_{i=1}^{K} \delta_{i}^{2} / K+\sum_{i=1}^{K} \sigma_{i}^{2} / K$

where $\mathrm{K}$ is the number of measured variables,

$\sigma \mathrm{i}, \mathrm{i}=1, \mathrm{~K}$ is the root mean square error (RMSE) of the $\mathrm{i}$-th variable measurement errors, and $\delta \mathrm{i}, \mathrm{i}=1, \mathrm{~K}$ is the fuzziness of the ith prediction.

Use of a priori information improves accuracy if the mean prediction error is less than the sum of the mean prediction fuzziness and the mean measurement error.

\section{B. Practical point of view}

Or in other words what gain could be achieved with a rather inaccurate prediction?

The typical measurement accuracy for the modern measurement instruments could be in the vicinity of $1-2 \%$. In this case, of say $2 \%$ measurement error, with the prediction fuzziness of say $20 \%$, which is rather high (for example, it might mean the prediction like "the measured variable has a value of around 10 units or actually somewhere roughly between 8 and 12 units", which in practical cases sounds like a very reasonable suggestion) could achieve the gain up to $44 \%$.

In complex geological measurements, accuracy is actually much lower. In a case of around $10 \%$ measurement errors, the same predictions as in the previous example could achieve $400 \%$ gain. One should understand that such values of gain could be achieved when the forecaster makes a correct prediction.

However, even in a case when a forecaster makes an error in his/her prediction, there could still be some gain. Speaking very roughly, in order to get any gain the error value should be smaller than a sum of the prediction fuzziness and the measurement error. This allows a forecaster to develop a strategy to avoid any lose. If a forecaster is confident about the prediction value, he/she may low prediction fuzziness and achieve a higher gain. However, when the confidence level 
decreases, the prediction fuzziness could be increased, which might lower the gain value but allow avoiding loses.

\section{CONCLUSION}

The problem of a priori expert's information use for improvement the measurement procedures quality and the estimates received may be considered as a fusion of information from different sources, which are characterized by different uncertainty degrees. This area attracts a particular attention over a last few years. The neuro-fuzzy methods and their applications in measurement information fusion, reported in the literature, have been classified into four groups according with the goal achieved with their introduction.

The problem has been formalized mathematically as an optimization problem with fuzzy constraints and the solution has been found for the normally distributed measurement results and a specific expert's information.

The properties of the modified estimates have been studied in comparison with the conventional ones. The modified estimates have been found more efficient under the condition when the prediction error does not overcome the sum of the average measurement error and the prediction fuzziness. The possible efficiency gain in geological applications was estimated. The procedures improving reliability of the modified estimates have been offered, which include recommendations to an expert on making predictions: when an expert's confidence in predicted value is high, a prediction fuzziness should be made low in order to achieve a high gain; however, with a decrease in confidence a fuzziness could be made wider in order to avoid the estimate corruption.

\section{REFERENCES}

[1] Dosser D.I., K.D.Crain, M.B.Baker, V.Kreinovich, M.G.Gerstenberger "Estimating uncertainties for geophysical tomography", Reliable Computing, vol.4, 1998, pp.241-268

[2] Russo F., G.Ramponi. "Fuzzy methods for multisensor data fusion . IEEE Transactions on Instrumentation and Measurement " vol. 43, No. 2, 1994, p. 288-294

[3] Butkiewicz B. "Position control system with fuzzy microprocessor AL220", Computational Intelligence Theory and Applications International Conference, 5th Fuzzy Days, Proceedings SpringerVerlag, Berlin, Germany 1997, pp. 74-81

[4] Bloch I. "Information combination operators for data fusion: a comparative review with classification", IEEE Transactions on Systems, Man and Cybernetics, part A, vol. 26. No.1, pp.52-67

[5] Qiao L, M.Sato, K.Abe, H.Takeda "Self-supervised learning Algorithm of Environment Recognition in Driving Vehicle", IEEE Transactions on Systems, Man and Cybernetics, part A, vol. 26, No. 6, 1996, pp. 843-850

[6] Naish N.D., E.A. Croft "ELSA: a multisensor integration architecture for industrial grading tasks", Mechatronics, vol. 10, No. 1-2, 2000, pp.19-51

[7] Kobayashi F., Arai F., Fukuda T., Shimojima K., Onoda M., Marui N. "Sensor fusion system using recurrent fuzzy inference", Journal of Intelligent \& Robotic Systems, vol. 23, No. 2-4, 1998, pp.201-216

[8] Rong L.,Ping W., Hu W.L. "A novel method for wine analysis based on sensor fusion technique" Sensors \& Actuators B-Chemical, v.66, No. $1-3,2000$, pp. 246-250
[9] Llobert E., Hines E.L., Gardner J.W., Franko S. "Non-destructive banana ripeness determination using a neural network-based electronic nose", Measurement Science \& Technology, v. 10, No. 6, 1999 , pp. 538-548

[10] Lazzerini B., Maggiore A., Marcelloni F. "Classification of odor samples from multisensor array using new linguistic fuzzy method." Electronics Letters, vol.34, No.23, 1998 , pp.2229-2231

[11] Lin C.T., Lee Y.C., Pu H.C. "Satellite sensor image classification using cascaded architecture of neural fuzzy network", IEEE Transactions on Geoscience \& Remote Sensing. vol. 38, No. 2, part 2, 2000, pp.1033-1043

[12] Belloir F., Huez R., Billat A. "A smart flat-coil eddy-current sensor for metal-tag recognition", Measurement Science \& Technology, vol.11, No.4, 2000, pp.367-374

[13] Reznik L. and G.Solopchenko "Use of a priori information on functional relations between measured quantities for improving accuracy of measurement", Measurement, 1985, vol. 3, No. 3, pp. 98106

[14] Reznik L., G. Solopchenko "Method of Complexing of Measurements", The USSR Patent: Bulletin of patents, 1986, No. 47 .

[15] Su L., C. Komata "Error correction using fuzzy logic in vehicle load measurement", In: 1997 First International Conference on KnowledgeBased Intelligent Electronic Systems Proceedings, KES '97, IEEE New York, NY, USA, 1997, vol. 2 pp 595-598

[16] Healy T.A., Kerr L.J., Larkin L.J. "Model based fuzzy logic sensor fault accommodation", Journal of Engineering for Gas Turbines \& Power-Transactions of the ASME. Vol. 120, No.3, 1998, pp.533-536

[17] Mauris G., Benoit E., Foulloy L. "Local measurement validation for an intelligent chirped-FM ultrasonic range sensor", IEEE Transactions on Instrumentation \& Measurement., v.49, No.4, 2000, pp. 835-839

[18] Dutta RK., Paul S., Chattopadhyay AB. "Fuzzy controlled backpropagation neural network for tool condition monitoring in face milling", International Journal of Production Research, v. 38, No.13, 2000, pp.2989-3010

[19] Kuo R.J."Multi-sensor integration for on-line tool wear estimation through artificial neural networks and fuzzy neural network", Engineering Applications of Artificial Intelligence, v.13, No.3, 2000, pp.249-261

[20] Reznik L.. "Fuzzy controllers", Newnes-Butterworth-Heinemann, Oxford-Boston, 1997, ISBN 0-7506-3429-4, 287 pp.

[21] Reznik L. and K.P. Dabke "Evaluation of uncertainty in measurement: A Proposal for application of intelligent methods" in $\mathrm{H}$. Imai/Ed. Measurement to Improve Quality of Life in the 21st Century, IMEKO -XV World Congress, June 13-18, 1999, Osaka, Japan, vol. II, p. $93-100$

[22] Reznik L. "What is intelligent measurement?" In: Computing with Words in Information /Intelligent Systems, L.A. Zadeh and J.Kacprzyk/Eds. Physica Verlag, Heidelberg - New York, 1999, pp. $78-89$ 